The spacecraft programme, however, would be of little value without the numerous ground-based rocket and balloon projects which will continue throughout the IMS period. Earthbased measurements of variations in the magnetic field can be used to monitor magnetic storms and plasma waves and even to predict changes in the magnetosphere. Correlating these measurements with satellite observations should indicate how changes in field strength affect the shape and dynamics of the magnetosphere. Many of the 39 countries which could not afford to be involved in the space programme are contributing to the IMS through a sophisticated world-wide network of ground observatories. Even at the beginning of 1976, more than 1,000 individual research projects were registered on the IMS computerised file.

Many different experimental programmes working towards the same end thus need coordinating. Occasionally, for example, two or more satellites may be in such a configuration that simultaneous observations would be very valuable. Similarly, satellite observations should sometimes be timed in with ground-based or rocket experiments. To coordinate observations, NASA helped set up an IMS Satellite Situation Centre at the beginning of the study at the World Data Center A for Rockets and Satellites at the Goddard Space Flight Center, Boulder, Colorado. Its function is to report on satellite positions, recommend periods of special interest, compile and up-date information on satellite experiments and answer inquiries on special projects, experiments and the whereabouts of satellites. Every month, the IMS Central Information Exchange Office, also at Boulder, produces an IMS news letter.

\section{Regaining ground}

Hopes for regaining the ground lost by the partial failure of Geos hang on the possibility of launching the second Geos satellite into geostationary orbit carly next year. The intention had been to place the first Geos in a geo-

International science (2)

\title{
WHO's show on the road
}

\section{Peter Collins describes the gestation of the World Health Organisation's tropical diseases progamme}

$T^{\text {HE temptation to launch global }}$ 1 campaigns against specific diseases or conditions of mankind is one the World Health Organisation (WHO) seems unable to resist. The most recent and from all appearances most successful is the smallpox eradication campaign. Pockets of the disease remain, principally in the horn of Africa, but the campaign's objectives are in sight in under ten years. By contrast, in spite of the malaria eradication campaign, which started in 1955, malaria has never been anywhere near eradication in much of Africa south of the Sahara, and is now increasing in other regions and countries where eradication was thought to have been achieved. Now WHO's thinking has turned towards a more broadly-based approach. It is well exemplified by the Special Programme on Research and Training in Tropical Diseases which is tackling six diseases (malaria, schistosomiasis, the filariases, trypanosomiasis, Leishmaniasis and leprosy) that affect some 700 million men, women and children.

Perhaps the greatest single constraint on the development of the tropics lies in the burden of disease borne by their human inhabitants. Probably one in every four people alive today is at risk from one or more of the six diseases with which the programme is concerned. The position worsens every year as population increases and the world's pharmaceutical industry stands back. No new drug against trypanosomiasis has appeared in the past 20 years; the only drugs available against the filariases have side effects so serious that their use for mass treatment is out of the question; and the drugs used for treating malaria are meeting with more and more resistance on the part of the parasites. Added to this is the increasing resistance of many of the vectors of these diseases to the chemicals used against them, and the many reservations about the use of DDT, still often the most effective weapon for insect vector control.

The sheer lack of scientific knowledge about many of these diseases is also a problem. Not enough is known about the biology of the parasite and the mechanisms by which it eludes the defences of the human host to allow intelligent intervention to be planned. But great advances in such fields as immunology have led to the evolution of a whole armoury of new weapons for the prevention, diagnosis and treatment of disease afflicting richer stationary orbit $36,000 \mathrm{~km}$ above the equator along a field line connecting the north and south auroral zones so that particles on their way to the aurora would pass through it. Groundbased experiments and rocket-borne observations of the aurora could then have been coordinated with experiments on board Geos.

But Geos is not in a geostationary orbit and coordinating observations is very difficult. It would have been impossible had the Geos ground control at Darmstadt not managed to manoeuvre the satellite into a doubly geosynchronous orbit shortly after the unsuccessful launch. Geos now passes through the neighbourhood of the planned geostationary orbit twice a day, meaning that coordinated observations are possible, but only for a very short time. If Geos 2 were launched next year, it could take the place of Geos 1. It would have to be launched on another Delta rocket, however, and ESA might decide to wait until the end of 1979, when it could be launched on Europe's launcher, Ariane.

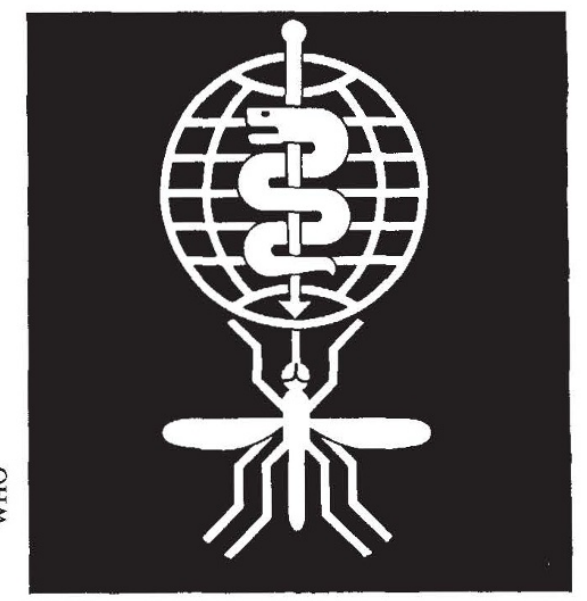

WHO anti-malaria emblem

countries, and the question is how these can be made available or adapted to help control the great endemic diseases of the tropical countrics.

\section{WHO's answer}

The present programme is WHO's answer to this question, but it originated within WHO from the conjunction of two quite separate idcas. In 1964 an expert group of immunologists and parasitologists was assembled for the first time in Geneva. Little common ground seemed to exist between these two disciplines thereto: the parasitologists considered that there was little if any immune response to the diseases with which they were concerned, and the immunologists had found ample scope, backed by adequate funds, in fields like cancer research. After this meeting, however, things began to change, and while the immunology of 
parasitic diseases was being examined, another initiative was being taken within WHO-the establishment in certain African countries of a network of biomedical research centres, eventually to be staffed and run by local personnel. The feeling was that only research by nationals of the countries concerned could achieve real control of tropical diseases.

It was from a 'meeting of minds' among senior staff involved in these two developments that the present programme evolved. By 1974 their ideas were ready for presentation to the World Health Assembly and subsequent examination by WHO's Advisory Committee on Medical Research (ACMR). The basic plan, further discussed with prospective participants, was developed in time for a major 'housekeeping' meeting in December last year. The pattern is that of a cooperative effort of donor and recipient governments, international agencies and specialised institutions, collaborating with and coordinated by WHO. A major part as co-sponsors will be played by the United Nations Development Programme (UNDP) and (though its precise rôle has yet to be determined) the World Bank. Because the programme involves a number of different WHO departments, a small Tropical Diseases Research Group (TDR) has been set up for overall scientific coordination and management. Progress will be reviewed annually by a Scientific and Technical Advisory Committee composed of scientists, selected on an individual basis, to make the best existing knowledge and experience available to those directing the programme. General oversight will be ensured by a Joint Consultative Board, expected to be convened later this year and designed to represent all the various groups of participants in the programme.

The programme is expected to cost between $\$ 10$ and $\$ 20$ million a year as presently foreseen. This money will come from governments through their development aid or technical assistance allocations, and from other sources of international funding. It is here that WHO might have anticipated some difficulty, for those concerned with development financing are naturally somewhat cautious when asked to support what is, admittedly, a vast experiment, at a time when funds for development projects are increasingly tight. Several different approaches to this type of financing are already apparent. The Scandinavians prefer to hand over funds directly, often seem. ing to take little interest in their management, although they may now be looking harder at the results than hitherto. The USA, on the other hand, appears to favour complete control of the programme funds, preferably by the World Bank. Britain's fairly neutral stand on that point is tempered by an insistence on continuing and critical evaluation of both the selection and progress of individual projects. Even at this early stage, several governments, Britain's included, have been highly critical of the potential of certain projects to which programme funds have been allocated by WHO. Intriguingly, it does seem that one problem is to find sufficient projects to absorb the funds already available, a situation that should reassure those who fear that the programme will prove to be yet another drain down which development funds are being uselessly poured.

\section{Two lines}

The two main lines of action that make up the agreed programme reflect its dual origin within WHO. The first is a vastly increased research effort directed at some of the main problems of the six selected diseases, and involving many of the ideas and techniques developed by biomedical research into the diseases of the industrialised, affluent world. Where possible, this research should be done where the diseases occur, and eventually (though this is not yet feasible) by nationals of the countries concerned. The second recognises that research, however successful, is of little use unless it can be properly exploited. This means training nationals of the tropical countries in the design, administration and application down to village level of whatever control system is finally evolved.

The underlying approach is not without precedent as far as WHO is concerned. Its programme for research into human reproduction was set up on much the same lines: a group of scientists was assembled, presented with certain ideas, asked if they thought a programme based on these ideas could be made to work, and urged to indicate the steps to be taken. This in itself was something rather new; biologists are not usually asked to do research with a definite end in view, and the human reproduction programme is one of WHO's current successes. That the system can work is also shown by the Scientific Working Group on the immunology of leprosy, which was set up in the same way and is already yielding results.

The parasitic diseases programme is of a different order of magnitude, though. Each of the scientific working groups is in itself an experiment directed to separate objectives. Apart from those dealing with leprosy, groups on filariasis, schistosomiasis and the immunology of malaria have been set up; others will be concerned with such broad fields as vector biology and the tissue culture of parasites. Each group examines its particular disease under headings like immunology, chemotherapy, epidemiology and training, and identifies desirable research projects and the institutes and individuals to tackle them. WHO envisages a network with a coordinating group at headquarters. On the one side are the 'collaborating institutes', the established centres of biomedical research, mostly located in the industrialised countries. Their collaboration will be sought for the more sophisticated research especially where new and special equipment and facilities are needed, and for training at the higher levels. On the other side are 'collaborating centres', at various levels from large university departments to small laboratories employing only a handful of qualified staff, and located mostly where the diseases are prevalent. It is in this group that the 'institution strengthening and training activities' of the programme will have first priority.

\section{No cutting}

As WHO sees it, there is no need for the programme to cut across the activities of the established institutions in the industrialise countries. But with too many people doing research into tropical diseases who have had little or no contact with those suffering from them, a properly coordinated operation of such a network should help progress. WHO would make a further point, that the programme offers opportunities that are, scientifically speaking, exciting, with the use of the new tools and high technology of modern biological science. The different approach to publicising the programme reflects this. In the past, pulilicity has been given to global programmes of public concern, with the aim of telling people about something being done for their welfarethe conquest or alleviation of rheumatism, for example (1977 is World Rheumatism Year). For the tropical diseases programme, WHO's aim is rather to interest the scientific community.

With the gradual run-down of the great colonial medical services, tropical disease research has been increasingly less popular. But now, the more people who know of this vast new programme and of its funding, the greater likelihood there is of interesting young research workers in the opportunities it has to offer. And at some $\$ 20$ million a year for, say, 20 years, it is not that expensive. A major pharmaceutical company can spend five times that annual sum in a single year, and some countries spend as much on cancer research as the total budget for this research on tropical diseases. 